\title{
INTIMIDAD Y VIGILANCIA EN LAS INVESTIGACIONES INTERNAS DERIVADAS DEL USO DE UN CANAL DE WHISTLEBLOWING
}

\author{
Danae Travé Sarradell \\ Graduate en Cuatrecasas
}

\begin{abstract}
El presente artículo analiza la obligación empresarial de investigar ante la existencia de posibles irregularidades en la organización, impuesta por la Directiva (UE) 2019/1937 y el artículo 31 bis del Código Penal junto con la doctrina aplicable a la protección del derecho a la intimidad en el trabajo, flexibilizada con la sentencia del Tribunal Europeo de Derechos Humanos López Ribalda II. La colisión de obligaciones generada requiere una respuesta que integre las diferentes normativas existentes y permita esclarecer bajo qué parámetros deberán llevarse a cabo las investigaciones internas en las empresas.
\end{abstract}

This paper analyses the surveillance obligations in the event of potential irregularities within the organization imposed to businesses by EU Directive 2019/1937 and Section 31 bis Criminal Code together with the applicable doctrine to employee's right to privacy -made more flexible in European Court of Human Right's ruling López Ribalda II-. The resulting clash of obligations requires an integrated response with common standard to set the limits of internal investigations within a company.

Title: Privacy and surveillance in internal investigations deriving from the use of whistleblowing channels.

Palabras clave: intimidad, poder de control y vigilancia, medios tecnológicos, López Ribalda II, sospechas fundadas, canales de denuncia, investigaciones internas, Directiva (UE) 2019/1937.

Key words: privacy, control and surveillance power, technological means, López Ribalda II, reasonable suspicion, whistleblowing channels, internal investigations, EU Directive $2019 / 1937$.

IUSLabor 1/2021, ISSN 1699-2938, p. 42-65

DOI. 10.31009/IUSLabor.2021.i01.02

Fecha envío: 8.11.2020 | Fecha aceptación: 20.01.2021 


\section{Sumario}

1. Introducción

2. Contexto normativo y evolución jurisprudencial del derecho a la intimidad en el trabajo

2.1. Estricta aplicación del juicio de proporcionalidad.

2.2. Información previa a los trabajadores sobre las posibilidades de uso de los medios y la importancia del concepto de la expectativa de privacidad

2.3. Flexibilización del requisito de la información previa y aplicación conjunta con el juicio de proporcionalidad

2.4. El cambio doctrinal establecido por el Tribunal Europeo de Derechos Humanos a partir de la sentencia Barbulescu II

2.5. La matización de la doctrina tras la sentencia López Ribalda II

3. Las denuncias internas por canales de whistleblowing como elemento detonante para el inicio de una investigación interna en la empresa

3.1. Concepto y regulación de los canales internos de denuncia como elemento clave en los planes de compliance

3.2. Las novedades introducidas por la Directiva (UE) 2019/1937

3.3. La calificación de las denuncias internas

3.4. La responsabilidad penal de la persona jurídica ante la omisión de vigilancia y control

4. Conclusiones

5. Bibliografía 


\section{Introducción}

El auge de la tecnología, la incorporación masiva de medios electrónicos en la sociedad y la híper-conectividad han transformado la vida en todos sus ámbitos y, por ende, también en el trabajo. Las novedades tecnológicas brindan a las empresas enormes oportunidades en términos de productividad y eficiencia, por lo que cada vez ocupan una posición más preeminente en los lugares de trabajo y su papel es fundamental para mantener el ritmo de la actividad empresarial y garantizar una posición competitiva en el mercado. Acortar distancias, facilitar comunicaciones, mejorar la seguridad y potenciar el rendimiento son algunas de las ventajas que la incorporación de medios tecnológicos puede brindar a las empresas.

Del mismo modo, desde la perspectiva del derecho del trabajo, la tecnología facilita al empresario la vigilancia y control de los trabajadores, pudiendo actuar de forma más rápida, accediendo a la información necesaria y en remoto. Así, escenarios que en la literatura se planteaban como distopías, ${ }^{1}$ podrían percibirse cada vez cercanos a la realidad en vista de la creciente transparencia de los trabajadores en el marco de la empresa. $^{2}$

Entre los múltiples motivos que pueden llevar al empresario a ejercitar su facultad de control o vigilancia, se encuentra el recibimiento de denuncias a través de los canales conocidos como whistleblowing channels. Resulta de especial relevancia en este punto la reciente Directiva (UE) 2019/1937 del Parlamento Europeo y del Consejo de 23 de octubre de 2019 relativa a la protección de las personas que informen sobre infracciones del Derecho de la Unión ("Directiva (UE) 2019/1937) que, a pesar de no haber sido todavía transpuesta por el legislador español, obligará a determinadas empresas a disponer de dichos canales internos de denuncia, así como a responder a las denuncias recibidas en un plazo máximo tasado, comunicándole al denunciante el resultado final de la investigación realizada. Del mismo modo, el artículo 31 bis de la Ley Orgánica 10/1995, de 23 de noviembre, del Código Penal ("Código Penal") impone el deber empresarial de vigilancia en la organización para garantizar una adecuada prevención ante la comisión de delitos.

El contenido de la Directiva (UE) 2019/1937 (así como del artículo 31 bis del Código Penal) es novedoso por cuanto introduce el carácter imperativo de las investigaciones

\footnotetext{
${ }^{1}$ Como en la obra de GEORGE ORWELL 1984, publicada en 1949.

2 BLASCO JOVER, Carolina, "Trabajadores "transparentes": la facultad fiscalizadora del empresario vs derechos fundamentales de los empleados (I)", Revista Internacional y Comparada de Relaciones Laborales y Derecho del Empleo, Adapt University Press, 2018, p. 31. Disponible en: http://ejcls.adapt.it/index.php/rlde_adapt/article/view/600.
} 
internas, a diferencia de la tradicional percepción de la vigilancia y control de los trabajadores como una facultad empresarial reconocida en el artículo 20.3 del Real Decreto Legislativo 2/2015, de 23 de octubre, por el que se aprueba el texto refundido de la Ley del Estatuto de los Trabajadores.

La vigilancia de los trabajadores en el marco de la relación laboral topa directamente con la protección de los derechos fundamentales, principalmente, con el derecho a la intimidad y a la vida privada, reconocido en el artículo 8 del Convenio para la Protección de los Derechos Humanos y de las Libertades Fundamentales, hecho en Roma el 4 de noviembre de 1959 (“CEDH”) y el artículo 18 de la Constitución Española, de 1978 ("CE"). Por ello, y especialmente en las últimas tres décadas, la privacidad en el centro de trabajo se ha convertido en una temática clave del derecho laboral comparado y cuya relevancia crece paralelamente al desarrollo y sofisticación de los medios tecnológicos al alcance de la empresa. ${ }^{3}$

Con el fin de garantizar un adecuado equilibrio entre los derechos e intereses de empleados y empleadores, y debido a la falta de actividad legislativa en esta materia, los tribunales han venido desarrollando los criterios que deben regir como medio de ponderación y las pautas que deben obedecer las empresas y los trabajadores. La existencia de tribunales supranacionales, concretamente el Tribunal Europeo de Derechos Humanos, ha resultado clave en este sentido. Así, la reciente sentencia López Ribalda II conforma, junto con Barbulescu II, la doctrina aplicable en la actualidad basada en la aplicación de seis criterios que vienen a describir el principio de proporcionalidad y un matizado requisito de información previa.

Las resoluciones emitidas en esta materia han analizado la protección del derecho a la vida privada como una contraposición entre derechos de los trabajadores e intereses empresariales. Sin embargo, la obligación de investigar que imponen los instrumentos normativos citados (la Directiva 2019/1937 y el artículo 31 bis del Código Penal) transforma esta dicotomía convirtiéndola en una contraposición de derechos y obligaciones. Por ello, resulta necesario establecer cuáles son las pautas que deben regir las investigaciones internas en estos casos y si la doctrina existente hasta la fecha permite matizaciones ante el carácter imperativo de la vigilancia empresarial.

Con base en lo expuesto, el objeto del presente estudio consiste en dilucidar, con un previo análisis de la normativa aplicable y de la evolución jurisprudencial en la materia, el papel de las denuncias emitidas por canales de whistleblowing en el marco de una investigación interna y hasta qué punto pueden considerarse un elemento que permita

\footnotetext{
${ }^{3}$ FLANAGAN, Julie. A., "Restricting electronic monitoring in the private workplace", Duke Law Journal, Vol. 93, 1994, P. 1257. Disponible en: https://scholarship.law.duke.edu/dlj/vol43/iss6/6/.
} 
flexibilizar o devaluar los criterios establecidos por la jurisprudencia en materia de vigilancia y control de los trabajadores.

El desarrollo de los distintos puntos permitirá demostrar la necesidad de regular conjuntamente el encaje entre el derecho a la intimidad y el control empresarial en el ámbito laboral en consonancia con la normativa aplicable a las investigaciones internas empresariales iniciadas a partir del uso de canales de denuncia. Todo ello, considerando además la posible responsabilidad penal de las personas jurídicas ante la falta de medidas de vigilancia y control suficientes para la prevención de delitos.

\section{Contexto normativo y evolución jurisprudencial del derecho a la intimidad en el trabajo}

La intimidad personal del trabajador, regulada en los artículos $8 \mathrm{CEDH}$ y $18 \mathrm{CE}$, constituye un derecho clave en el marco de la relación laboral y se plantea como un límite común, incluso en derecho comparado, ante la utilización de los medios de control del empresario. ${ }^{4}$

En virtud de la eficacia horizontal de los derechos fundamentales, los derechos reconocidos en los artículos $8 \mathrm{CEDH}$ y $18 \mathrm{CE}$ son aplicables de forma directa al conjunto de la ciudadanía en todos los ámbitos de la vida y por ende también en el marco de las relaciones laborales. ${ }^{5}$ Sin embargo, en la medida en que estas se desenvuelven en el seno de una organización, el ejercicio de derechos por parte de los trabajadores admite ciertas limitaciones o sacrificios cuando entra en colisión con otros derechos reconocidos constitucional o legalmente a los empresarios. ${ }^{6}$ En este sentido, destacan el derecho a la propiedad (artículo $33 \mathrm{CE}$ ), el derecho a la libertad de empresa (artículo $38 \mathrm{CE}$ ) y el poder de vigilancia y control empresarial, que reconoce al empresario la facultad de adoptar "las medidas que estime más oportunas de vigilancia y control para verificar el cumplimiento por el trabajador de sus obligaciones y deberes laborales" (artículo 20.3 ET).

\footnotetext{
${ }^{4}$ MARTINEZ FONS, Daniel, "El poder de control del empresario en la relación laboral", Madrid, Consejo Económico Social, 2002, P. 77.

${ }^{5}$ Tal y como afirman, entre otras, las SSTC 88/1985, 99/1994, 90/1997 y 204/1997: "la celebración de un contrato de trabajo no implica la privación en modo alguno para una de las partes, el trabajador, de los derechos que la Constitución le reconoce como ciudadano".

${ }^{6}$ STC (Sala Primera) 90/1997, de 6 de mayo.
} 
Así, sin perder de vista el principio de indisponibilidad de los derechos laborales (artículo 3.5 ET), ${ }^{7}$ la inserción de los trabajadores en la vida laboral permite determinadas modulaciones ${ }^{8}$ de sus derechos en conjunción con los requerimientos razonables de la organización productiva en la que se integran, con la finalidad de alcanzar un adecuado equilibrio entre los mismos. ${ }^{9}$

A pesar de la habilitación tanto del CEDH como de la CE para regular esta materia legislativamente, más allá de previsiones convencionales o de códigos de conducta, los parámetros que deben regir la actividad empresarial para respetar el derecho a la intimidad y vida privada de sus trabajadores no se encuentran desarrollados en leyes. Ante dicha escasez normativa los órganos judiciales han sido los encargados de establecer las pautas y criterios aplicables en la materia, ejerciendo una función cuasi legislativa. ${ }^{10}$ Por ello, con el fin de conocer cuáles son los criterios de aplicación, resulta clave analizar la evolución de los estándares utilizados por la jurisprudencia, tanto del Tribunal Europeo de Derechos Humanos como de los tribunales nacionales.

El punto de partida de dicho análisis jurisprudencial se encuentra en la facultad de control del empresario que, a su vez, pivota sobre la existencia de una relación laboral entre las partes y el poder patrimonial del empresario sobre de los medios tecnológicos utilizados por los trabajadores.

Así, el artículo 20.3 ET constituye ${ }^{11}$ la principal fuente de legitimación del poder de control del empresario. Su relevancia viene además justificada a) por un lado, puesto que

\footnotetext{
${ }^{7}$ En virtud de este principio serán nulos aquellos actos de los trabajadores que impliquen la abdicación de sus derechos (STS 27 de abril 1999 (rec. 4985/1997) y STSJ Cataluña de 12 de febrero 2004 (rec. 498/2001), entre otras.

8 "Estas limitaciones o modulaciones tienen que ser las indispensables y estrictamente necesarias para satisfacer un interés empresarial merecedor de tutela y protección”. STC (Sala Primera) 98/2000, de 10 de abril.

${ }^{9}$ Debido a la dificultad que conlleva dicho ejercicio de ponderación, existen incluso opiniones que entienden que alcanzar el verdadero término medio es imposible, pues los valores no tienen el mismo peso, por lo que el equilibrio se encontrará siempre en un punto más próximo a un extremo que a otro de la balanza. Así se manifiesta en MERCADER UGUINA, Jesús, "El impacto del uso de dispositivos tecnológicos y Derechos Fundamentales: nuevas respuestas para un tiempo nuevo", IUSLabor, núm. 2, 2018, p. 2.

${ }^{10}$ SALA FRANCO, Tomás, "El derecho a la intimidad y a la propia imagen y las nuevas tecnologías de control laboral" en BORRAJO DACRUZ, Efrén. (coord.) Trabajo y libertades públicas, Madrid, Wolkers Kluwer (La Ley), 1999, P. 205.

${ }^{11}$ En un momento jurisprudencial más embrionario, a raíz de la creciente introducción de los medios electrónicos y la tolerancia empresarial hacia el uso privativo de los mismos, los tribunales rigieron las actuaciones de vigilancia del empresario con base en el artículo 18 ET y sus garantías (entre otras, la STSJ Andalucía/Málaga 25 de febrero 2000 (rec. 2207/1999) o la STSJ Cantabria 20 de febrero 2004 (rec.
} 
es la materialización en la normativa laboral del principio de libertad de empresa y b) por otro, debido a que el artículo 20.3 ET sitúa el límite del control empresarial en la dignidad de los trabajadores, siendo el derecho a la intimidad y a la vida privada un derecho estrictamente vinculado a la personalidad y una manifestación de la dignidad. ${ }^{12}$

Sobre esta base, la evolución de la jurisprudencia en materia de protección del derecho a la intimidad en el trabajo puede dividirse en cinco etapas, caracterizadas por los elementos y resoluciones clave que se analizarán a continuación y cuya combinación y encaje permite construir la doctrina aplicable a la materia en la actualidad.

\subsection{Estricta aplicación del juicio de proporcionalidad}

En una primera etapa jurisprudencial, ${ }^{13}$ el juicio de proporcionalidad desarrollado por el Tribunal Constitucional ${ }^{14}$ fue el criterio utilizado para resolver la ponderación de derechos e intereses a efectos de garantizar la intimidad en el ámbito laboral. Así se observa en las sentencias SSTC (Sala Primera) 98/2000, de 10 de abril, ( caso La Toja) y (Sala Primera) 186/2000, de 10 de julio, (caso Ensidesa), manifestándose en esta última que "la constitucionalidad de cualquier medida restrictiva de derechos fundamentales viene determinada por la estricta observancia del principio de proporcionalidad" (FJ $\left.6^{\circ}\right)$.

Entre los elementos considerados para desarrollar dicho ejercicio de ponderación, la existencia de sospechas razonables y fundadas sobre una posible irregularidad por parte del empresario constituyó un elemento determinante que justifica además la diferencia en

47/2004). Así se expone en RODRÍGUEZ ESCANCIANO, Susana, Poder de control empresarial, sistemas tecnológicos y derechos fundamentales de los trabajadores. Valencia, Tirant lo Blanch, 2015, pp 44 y ss.

${ }^{12}$ Entre otras: SSTS (Sala Segunda) 117/1994, de 25 de abril; (Sala Primera) 12/2012, de 30 de enero; (Sala Segunda) 167/2013, 7 de octubre.

${ }^{13}$ La doctrina jurisprudencial en esta materia puede clasificarse en tres periodos, en función de cuál ha sido el elemento o criterio predominante: un primer periodo del principio de proporcionalidad -representado por las SSTC 98/2000 (caso La Toja) y 189/2000 (caso Ensidesa)-, un segundo periodo de exigencia estricta de la información previa y un tercer periodo de flexibilidad. Así se expone en: HENRÍQUEZ TRILLERÍA, Sebastián, "Protección de datos, videovigilancia laboral y doctrina de la Sentencia López Ribalda II: un peligroso camino hacia la degradación de la obligación de información” IUSLabor, núm. 3, 2019, pp. 5962. Disponible en: https://www.upf.edu/web/iuslabor/3/2019.

${ }^{14}$ El juicio de proporcionalidad presenta un triple contenido: i) el juicio de idoneidad o capacidad de conseguir el objetivo propuesto, ii) el juicio de necesidad o la inexistencia de otra medida más moderada que permita alcanzar el objetivo y iii) el juicio de proporcionalidad en sentido estricto, es decir, determinar si la medida generar más beneficios o ventajas para el interés general que perjuicios sobre otros bienes o valores en conflicto. Así se establece en SEMPERE NAVARRO, Antonio V., "Un apunte sobre la grabación mediante cámaras (Al hilo de la STS-CIV 600/2019 de 7 de noviembre)", Revista Aranzadi Doctrinal, núm. 2, 2020. 
el fallo de ambas resoluciones. Ya en esta primera etapa, en el caso Ensidesa, el Tribunal Constitucional entendió que la clave para considerar proporcionada y legitimar la vigilancia empresarial secreta o desinformada era la existencia de sospechas previas por parte del empresario a raíz de las irregularidades contables observadas en el centro de trabajo.

\subsection{Información previa a los trabajadores sobre las posibilidades de uso de los medios y la importancia del concepto de la expectativa de privacidad}

Tras el pronunciamiento por parte del Tribunal Europeo de Derechos Humanos en el caso Copland $^{15}$ (doctrina ya anticipada años atrás por el caso Halford ${ }^{16}$ ), a partir de 2007 se inició una segunda etapa jurisprudencial, donde los tribunales nacionales incorporaron la obligación de informar, con carácter previo al ejercicio de control empresarial, de las posibles injerencias por parte del empleador sobre los medios tecnológicos puestos a disposición de sus trabajadores para eliminar la expectativa de privacidad que pudiera tener el trabajador sobre ellos y legitimar la actuación de control. ${ }^{17}$

A pesar de no tratarse de un posicionamiento unánime, ${ }^{18}$ en algunas de sus resoluciones el Tribunal Supremo otorgó carácter preferente incluso suficiente al requisito de información previa. Si una vez recibida dicha información el trabajador seguía utilizando los medios para fines personales, su uso no estaría amparado por garantía alguna de confidencialidad, ${ }^{19}$ avalándose incluso en algunas resoluciones la existencia de políticas empresariales con prohibiciones absolutas de uso personal de los medios puestos a disposición del trabajador. ${ }^{20}$

La gran novedad jurisprudencial en la interpretación del requisito de información previa y el referente de su estricta aplicación llegó de la mano de la STC (Sala Primera) 29/2013

\footnotetext{
${ }^{15}$ STEDH (Sección 4a) de 3 de abril 2007 (demanda núm.62617/2000), Copland c. Reino Unido

${ }^{16}$ STEDH de 25 de junio 1997 (demanda núm. 20605/1992), Halford c. Reino Unido.

${ }^{17}$ SSTS (Sala de lo Social, Sección 1a) de 26 de septiembre 2007 (rec. 966/2006) y 8 de marzo 2011 (rec. 1826/2010).

${ }^{18}$ Entre otras, las SSTSJ Cataluña (Sala de lo Social, Sección 1a) de 28 de enero 2011 (rec. 4293/2011) que posteriormente alcanzaría el Tribunal Europeo de Derechos Humanos en el caso López Ribalda- y Castilla y León, Burgos (Sala de lo Social, Sección 1ª) de 10 de mayo 2006 (rec. 1249/2005), siguieron aplicando estrictamente el principio de proporcionalidad.

${ }^{19}$ BLASCO JOVER, Carolina, op. cit., p. 35.

${ }^{20}$ La STS (Sala de lo Social, Sección 1a) de 6 de octubre 2011 (rec. 4053/2010) afirmaba: "si no hay derecho a utilizar el ordenador para usos personales, no habrá tampoco derecho para hacerlo en unas condiciones que impongan un respeto a la intimidad o al secreto de las comunicaciones, porque, al no existir una situación de tolerancia del uso personal, tampoco existe ya una expectativa razonable de intimidad y porque, si el uso personal es ilícito, no puede exigirse al empresario que lo soporte y que además se abstenga de controlarlo".
} 
de 11 de febrero (caso Universidad de Sevilla). ${ }^{21}$ En su resolución, el Tribunal Constitucional consideró que la videovigilancia empresarial llevada a cabo vulneraba los derechos del trabajador (concretamente la protección constitucional de los datos personales, con base en el artículo $18.4 \mathrm{CE})^{22}$ por cuanto no se había cumplido correctamente con el requisito de información previa. Para ello, la información debía ser "previa y expresa, precisa, clara e inequívoca a los trabajadores de la finalidad de control de la actividad laboral" y "debía concretar las características y el alcance del tratamiento de datos que iba a realizarse, esto es, en qué casos las grabaciones podían ser examinadas, durante cuánto tiempo y con qué propósitos, explicitando muy particularmente que podían utilizarse para la imposición de sanciones disciplinarias por incumplimientos del contrato de trabajo" (FJ $\left.8^{\circ}\right)$.

Por lo tanto, con esta resolución (si bien el voto particular de la sentencia reafirmaba la importancia de aplicar el juicio de proporcionalidad y dejaba entrever la posición que posteriormente se adoptaría en el caso Bershka), la información por parte de la empresa se reafirmó como un requisito previo a cualquier otro análisis, pero, sobre todo, fue novedosa por cuanto determinó cuál deberá ser el contenido de dicha información. No toda información sería válida para neutralizar una posible vulneración de derechos fundamentales y, en los supuestos de videovigilancia, en ningún caso se podrá entender satisfecho el requisito mediante la presencia de distintivos anunciando la instalación de cámaras.

Sin embargo, la aplicación del principio de información previa en sentido estricto tuvo un recorrido limitado y la STC 29/2013 una aceptación matizada. ${ }^{23}$, La evolución de la jurisprudencia ${ }^{24}$ se posicionó más cercana a una aplicación conjunta de ambos criterios

\footnotetext{
${ }^{21}$ HENRÍQUEZ TRILLERÍA, Sebastián, op. cit., pp. 62-63.

${ }^{22}$ A pesar de enmarcarse en el artículo 18.4 CE, esta resolución resulta aplicable al concepto de la intimidad y vida privada de los trabajadores. Tal y como manifiesta la doctrina del pleno del Tribunal Constitucional en la STC (Pleno) 29/2000, de 30 de noviembre (que aplicó a su vez la STC 29/2013 en el caso Universidad de Sevilla), el contenido del artículo 18.4 CE plasma en el ordenamiento jurídico un ámbito de protección más específico a los datos personales que el del artículo 18.1 CE (FJ $4^{\circ}$ ), pero no excluyente, puesto que comparten "el objetivo de ofrecer una eficaz protección constitucional de la vida personal y familiar" (FJ $5^{\circ}$ ). Por lo tanto, el artículo 18.4 CE, aunque con un contenido más amplio (FJ $6^{\circ}$ ), tiene entre sus fines la protección de la intimidad y la vida que protege el artículo $18 \mathrm{CE}$ y el artículo $8 \mathrm{CEDH}$.

${ }^{23}$ GARCÍA RUBIO, Ma Amparo, "Nueva doctrina constitucional sobre videovigilancia y protección de datos personales". ELDERECHO.COM [en línea], 17 de julio de 2016 (https://elderecho.com/nuevadoctrina-constitucional-sobre-videovigilancia-laboral-y-proteccion-de-datos-personales; 8 de noviembre de 2020).

${ }^{24}$ Por un lado, la STS (Sala de lo Social, Sección $1^{\text {a }}$ ) 13 de mayo 2014 (rec. 1685/2013) (FJ 2º y la STSJ Castilla-La Macha (Sala de lo Social, Sección 1 ${ }^{\mathrm{a}}$ ) 11 de febrero 2016 (rec. 1714/2015) (FJ $7^{\circ}$ ) resolvieron en la línea de la STC 29/2013 y consideraron que el uso no consentido ni previamente informado de videocámaras instaladas con carácter permanente y utilizadas para un fin distinto del expresamente
} 
que permitiera considerar los elementos concretos que contextualizan cada caso y que evolucionaría hacia una creciente flexibilización del requisito de información.

\subsection{Flexibilización del requisito de la información previa y aplicación conjunta con el juicio de proporcionalidad}

En 2016, el Pleno del Tribunal Constitucional resolvió en su STC 39/2016, de 3 de marzo, el caso conocido como caso Bershka. El supuesto de hecho enjuiciado consistía en la instalación de cámaras enfocando a las cajas de una tienda debido a la sospecha empresarial de sustracción de dinero. El Tribunal, cambiando el criterio establecido en 2013, consideró que no existía vulneración de derechos puesto que la injerencia superaba el juicio de proporcionalidad en tanto que la existencia de sospechas fundadas conllevaría la frustración de la finalidad de la medida en caso de informar de ella a los trabajadores y la colocación de las cámaras y la duración de la grabación eran concretos y limitados en el tiempo. En cuanto al requisito de la información previa, este se consideró satisfecho mediante la colocación de un distintivo informativo indicando que el local estaba video vigilado.

La doctrina Berskha, acogida por los tribuales nacionales, ${ }^{25}$ representó una gran flexibilización del requisito de información previa respecto al estándar establecido en el caso Universidad de Sevilla e introdujo de la mano del Tribunal Constitucional la combinación de los principios de información y proporcionalidad, sirviéndose de este último y de la necesidad de garantizar la efectividad de la investigación realizada en la empresa, para rebajar las exigencias del deber de información. ${ }^{26}$ En definitiva, la STC 39/2016 permitió legitimar aquellas medidas empresariales que (a) contaran con algún tipo de información para el trabajador, aunque esta no fuera expresa o completa y (b) superaran el juicio de proporcionalidad, especialmente si existían sospechas previas que

señalado constituía una vulneración del derecho protegido por el artículo $18.4 \mathrm{CE}$, a la protección de datos personales. Por otro lado, en cambio, hubo pronunciamientos como las SSTSJ Madrid (Sala de lo Social, Sección 6 ${ }^{\text {a }} 9$ de febrero 2015 (rec. 886/2014) (FJ 4º), Cataluña (Sala de lo Social, Sección 1a) 1 de julio 2013 (rec.1804/2013) (FJ 3 ${ }^{\circ}$ ) y Castilla y León/Valladolid (Sala de lo Social, Sección 1 ${ }^{\text {a }}$ ) 1 de octubre 2015 (rec. 1940/2013) (FJ $1^{\circ}$ ) que entendieron que no procedía aplicar el razonamiento del caso Universidad de Sevilla por cuanto el supuesto de hecho no correspondía a la instalación de un sistema de grabación con carácter preventivo y anterior a cualquier incumplimiento laboral, sino que se trataba de una grabación episódica y de breve duración realizada tras la existencia de sospechas o irregularidades y que respetaba por lo tanto el principio de proporcionalidad.

25 Entre otras, las SSTS (Sala de lo Social) 7 de julio 2016 (rec. 3233/2014), 31 de enero 2017 (rec. 3331/2015), 1 de febrero 2017 (rec. 3262/2015) (caso Plana y Dieguez SL) y 2 de febrero 2017 (rec. 554/2016) (caso DIR).

${ }^{26}$ Es a partir de esta sentencia donde puede entenderse que empieza una etapa de flexibilización entre los criterios del juicio de proporcionalidad y la información previa utilizados por la jurisprudencia. Así se manifiesta en: HENRÍQUEZ TRILLERÍA, Sebastián, op. cit, p. 64 
permitieran justificar que no se trataba de una injerencia arbitraria. Esta posición, se mantendría tanto a nivel nacional como por parte del Tribunal Europeo de Derechos Humanos hasta la llegada de la resolución de la Gran Sala del caso Barbulescu, en septiembre de 2017.

\subsection{El cambio doctrinal establecido por el Tribunal Europeo de Derechos Humanos a partir de la sentencia Barbulescu II}

Tras la resolución emitida por la Sección $4^{\mathrm{a}}$ del Tribunal Europeo de Derechos Humanos en 2016 en la primera sentencia del caso Barbulescu, ${ }^{27}$ que confirmaba el posicionamiento adoptado por los tribunales nacionales desde la STC 39/2016, la doctrina en materia de protección del derecho a la intimidad en el trabajo dio un nuevo giro cuando el caso alcanzó la Gran Sala.

El supuesto de hecho analizado versaba sobre la interceptación y transcripción por parte del empresario de mensajes electrónicos, instantáneos y personales emitidos por un trabajador desde el lugar de trabajo, constando prohibición expresa de uso personal de los medios electrónicos de la empresa.

La sentencia Barbulescu II $^{28}$ consideró que sí existía vulneración del artículo 8 CEDH por cuanto no se había informado al trabajador de la posibilidad de que sus conversaciones fueran supervisadas (Ap. 77) y la empresa había accedido al contenido de las comunicaciones sin mediar causa concreta o sospecha fundada (Ap. 78).

En línea con este posicionamiento, en el apartado 120 de la resolución, se definieron los seis factores que deberían cumplirse (de forma cumulativa) para considerar la licitud o no del control empresarial, en virtud del artículo $8 \mathrm{CEDH}$ :

- El cumplimiento del requisito de información previa acerca de la posibilidad de tomar control del contenido protegido.

- El alcance del control o el grado de intromisión sobre la información personal protegida por el derecho a la intimidad y a la vida privada. En este sentido, se distingue entre el control del flujo de comunicaciones o su contenido, entre el acceso a la totalidad de las comunicaciones o a una parte, si la medida ha sido limitada en el tiempo o no, así como el número de personas que han tenido acceso a la información encontrada.

\footnotetext{
${ }^{27}$ STEDH (Sección 4 $4^{\mathrm{a}}$ ) 12 de enero 2016 (demanda núm. 61496/2008), Barbulescu c. Rumania.

${ }^{28}$ STEDH (Gran Sala) 5 de septiembre 2017 (demanda núm. 61496/2008) Barbulescu c. Rumania.
} 
- La existencia de motivos legítimos que justifiquen la vigilancia y el acceso al contenido protegido, entiendo que la existencia de sospechas fundadas reforzaría la legitimación de la injerencia empresarial.

- La posibilidad de haber utilizado alternativas menos intrusivas que el acceso al contenido protegido.

- El impacto o las consecuencias de la medida ejercitada, es decir, si los resultados se utilizaron para la finalidad perseguida por la medida, o para otros fines.

- La existencia de garantías para el trabajador, que impidieran que el empleador tuviera acceso al contenido de las comunicaciones sin que al empleado le hubiera sido previamente notificada tal eventualidad.

Así, la resolución de la Gran Sala en Barbulescu II desarrolla en sus seis puntos los criterios de información previa y proporcionalidad combinándolos de forma cumulativa, sin que se permitan deficiencias en el cumplimiento de ninguno de ellos, lo que convierte en muy estricto el umbral de la protección del derecho a la intimidad. El resultado es una doctrina novedosa que combina un posicionamiento estricto respecto a la información proporcionada a los trabajadores, similar al planteado en la STC 29/2013, junto con la aplicación del principio de proporcionalidad, con un cierto paralelismo al planteado en la STC 39/2016.

\subsection{La matización de la doctrina tras la sentencia López Ribalda II}

En enero de 2018, recién consolidada la doctrina Barbuslescu, la Sección $3^{\text {a }}$ del Tribunal Europeo de Derechos Humanos resolvió la primera sentencia del caso López Ribalda, ${ }^{29}$ un supuesto de videovigilancia encubierta de trabajadores de una cadena de supermercados. La empresa había venido identificando importantes y crecientes pérdidas por lo que inició una investigación interna para arrojar luz sobre las mismas. Así, se instalaron en el centro de trabajo cámaras de vigilancia de dos tipos: cámaras visibles que enfocaban las entradas y salidas del supermercado y sobre las cuales informó a los trabajadores y cámaras ocultas que apuntaban a las cajas, sobre las cuales no informó a los trabajadores. Tras diez días desde la instalación de las cámaras, se informó al delegado sindical que las imágenes habían capturado robos en las cajas por parte de varios empleados, que fueron despedidos.

\footnotetext{
${ }^{29}$ STEDH (Sección 3 $3^{\text {a }} 9$ de enero 2018 (demandas núm. 1874/2013 y 8567/2013), López Ribalda y otros c. España.
} 
A diferencia de lo considerado por los tribunales nacionales, ${ }^{30}$ el Tribunal Europeo de Derechos Humanos entendió que había existido una vulneración de derechos conforme al artículo $8 \mathrm{CEDH}$ puesto que faltaba la concurrencia de uno de los requisitos exigidos por Barbulescu: el requisito de la información. Además, la existencia de información parcial sesgaba especialmente la expectativa de los trabajadores, que no podían razonablemente esperar que pudieran estar vigilados en otras áreas de la tienda sin haber sido informados previamente (Ap. 93). Así, la sentencia López Ribalda I extendió los criterios establecidos en Barbulescu II a los casos de videovigilancia de trabajadores y destacó de nuevo la relevancia del cumplimiento de todos los requisitos de forma estricta y cumulativa.

Sin embargo, en octubre de 2019, la flexibilidad se impuso nuevamente con la sentencia López Ribalda II. ${ }^{31}$ Casi dos años después de la sentencia de la Sección $3^{\text {a }}$ la Gran Sala del Tribunal Europeo de Derechos Humanos reformuló de nuevo la doctrina en materia de protección del derecho a la intimidad y vida privada de los trabajadores y consideró que no había existido vulneración del artículo $8 \mathrm{CEDH}$ en dicho supuesto de videovigilancia encubierta.

Tras analizar el cumplimiento de los seis criterios establecidos en Barbulescu (Ap. 118 a 137), la sentencia resolvió flexibilizando el requisito que precisamente había ocasionado mayor discordia: la información previa. Así, la resolución concluyó que el conjunto de criterios de la doctrina Barbulescu no deberán interpretarse de forma totalmente rígida y que la ausencia o el deficiente cumplimiento de uno de ellos no conllevará automáticamente la vulneración del derecho a la intimidad y vida privada.

El requisito de información previa, si bien es muy importante en el marco de la configuración de un equilibrio entre la intimidad de los trabajadores y el poder de control empresarial, constituye únicamente uno de los criterios a considerar. Por lo tanto, no será suficiente para legitimar una medida (debiendo necesariamente combinarse con el principio de proporcionalidad) ni tampoco imprescindible, especialmente en aquellos supuestos donde existan sospechas fundadas o motivos que justifiquen la desinformación, bajo el riesgo de frustrar la viabilidad de la medida. En otras palabras, el Tribunal Europeo de Derechos Humanos aceptó la legalidad en determinados supuestos de la utilización de cámaras ocultas por parte de los empresarios en el lugar de trabajo cuando de ello pudiera depender garantizar el objeto de investigación. En estos casos, si así lo exigen la

\footnotetext{
${ }^{30}$ Tanto el Juzgado de lo Social de Granollers como el TSJ de Cataluña, en las SSTSJ Cataluña (Sala de lo Social, Sección 1a) 28 de enero 2011 (rec. 4293/2010) y 24 de febrero 2011 (rec. 4294/2010), consideraron que no se habían vulnerado los derechos fundamentales de los trabajadores puesto que la medida implementada por la empresa era necesaria, idónea y proporcionada.

${ }^{31}$ STEDH (Gran Sala) 17 de octubre 2019 (núm. 1874/2013 y 8567/2013), López Ribalda y otros c. España.
} 
necesidad y la finalidad de la medida por la existencia de sospechas fundadas, podrá aceptarse la existencia de una información genérica.

La combinación de la heterogeneidad jurisprudencial existente y de los diferentes criterios expuestos constituye la doctrina aplicable en la actualidad en materia de protección del derecho a la intimidad y a la vida privada en el marco de las relaciones laborales. Sin embargo, las resoluciones analizadas contemplan la acción de vigilancia empresarial como una facultad que les otorga el artículo 20.3 ET al servicio de sus intereses y el ejercicio de ponderación como una contraposición de derechos de los trabajadores e intereses empresariales.

Como apuntábamos al inicio, la introducción de instrumentos normativos como la Directiva (UE) 2019/1937 y el artículo 31 bis del Código Penal transforman la facultad de vigilancia empresarial en una obligación de investigar, que deberá encajarse junto a la doctrina existente en materia de protección de la intimidad, tal y como se analiza en el siguiente apartado.

\section{Las denuncias internas por canales de whistleblowing como elemento detonante para el inicio de una investigación interna en la empresa}

El ejercicio de la facultad de control o vigilancia empresarial puede venir motivado por múltiples realidades. Desde la existencia de un hallazgo casual por parte del empresario hasta la implementación de una investigación interna a raíz de una causa o sospecha previa. Precisamente en este último escenario es en el que se enmarcan las investigaciones que se inician tras el recibimiento de denuncias a través de los canales de denuncia conocidos como whistleblowing channels.

Una vez identificados los criterios que rigen las decisiones judiciales en materia de protección de la intimidad de los trabajadores ante una posible injerencia empresarial, procederemos en el presente apartado a analizar la aplicabilidad y adaptación de dicha doctrina en las investigaciones iniciadas a raíz de una denuncia emitida a través de un canal de whistleblowing.

A tal efecto, se analizará el concepto e importancia de los canales de denuncia, así como su regulación y las novedades que presenta la Directiva (UE) 2019/1937. A continuación, se valorará si las denuncias internas pueden calificarse como "sospechas fundadas" en el marco de una investigación interna y, en su caso, cuándo. Por último, se hará una breve mención al papel que juega en este contexto la posible responsabilidad penal de las personas jurídicas según lo dispuesto en el artículo 31 bis del Código Penal. 
3.1. Concepto y regulación de los canales internos de denuncia como elementos clave en los planes de compliance

Los canales de denuncia o whistleblowing channels son sistemas internos de comunicación entre la empresa y los empleados (u opcionalmente terceros como socios, proveedores o clientes), para que estos puedan informar sobre actos delictivos, incumplimientos o irregularidades de los que hayan tenido conocimiento y que sean contrarios tanto a la normativa interna de la empresa como externa, es decir, a la legalidad. ${ }^{32}$ Dichos canales, cuyo nombre proviene del uso del silbato de los policías británicos ante el apercibimiento de la comisión de un delito (to blow the whistle), ${ }^{33}$ constituyen un elemento fundamental en el marco de los planes de cumplimiento normativo o compliance, de origen anglosajón e importancia creciente en la actualidad. En el momento en que una denuncia entra en el canal, el programa de cumplimiento deberá tener previsto el funcionamiento y activación de un engranaje que permita implementar la debida investigación interna en la empresa.

Los planes de compliance que elaboran las empresas son, en definitiva, una manifestación más del poder de dirección y control empresarial que otorga el artículo 20 ET, por lo que impactan en la relación laboral y su cumplimiento puede ser exigible a los trabajadores en la medida en que no excedan los límites de la potestad de control y dirección empresarial.

El origen de la regulación de los sistemas de denuncias internas se encuentra en Estados Unidos, con la Whistleblowing Protection Act de 1998 destinada al ámbitode los empleados públicos- y la Sarbanes-Oxley Act de 2002 en el marco de las auditorias de sociedades cotizadas-. ${ }^{34}$

En el ámbito europeo las primeras manifestaciones de la regulación de canales de denuncia se encuentran en materias específicas: (i) el Dictamen 1/2006 sobre la aplicación de las normas de la UE relativas a la protección de datos a programas internos de denuncia de irregularidades en el campo financiero y contable y (ii) el Reglamento (UE) $\mathrm{n}^{\mathrm{o}}$

${ }^{32}$ MARTín POLVORINOS, César, "Guía de implementación de compliance para Pymes". World Compliance Association., 2019, P. 110.2 Disponible en: http://www.worldcomplianceassociation.com/documentacion/Guia_Compliance_web_v.02.pdf.

${ }^{33}$ PÉREZ TREVIÑO, José Luís, "Whistleblowing”, Eunomía. Revista en cultura de la legalidad, núm. 14, 2018, P. 286, que aborda ampliamente el origen y concepto del término whistleblowing.

${ }^{34}$ LOUSADA AROCHENA, José Fernando, "Sistemas de denuncias internas (Whistleblowing) y derechos fundamentales en el trabajo, Trabajo y Derecho, núm. 52/2019, p. 2. 
596/2014, del Parlamento Europeo y del Consejo sobre abuso de Mercado, de 2 de julio de 2014, que incluía la protección adecuada de los trabajadores que, en el marco de su actividad laboral, comunicasen infracciones o fuesen acusadas de cometerlas, frente a represalias de discriminación y otros tipos de trato injusto (artículo 32). ${ }^{35}$

A nivel nacional, los sistemas de denuncias internas fueron introducidos en la vida jurídica española en forma de recomendación mediante el Código de Buen Gobierno de las Sociedades Cotizadas de la Comisión de Mercado de Valores, para que los empleados pudieran comunicar las irregularidades financieras y contables observadas en el seno de la empresa. ${ }^{36}$

El común denominador de los distintos instrumentos normativos enunciados se encuentra en la protección del denunciante frente a represalias como medio necesario para garantizar la eficacia de los sistemas de denuncias internas.

Por su parte, la Ley Orgánica 3/2018, de 5 de diciembre, de Protección de Datos Personales y Garantía de los Derechos Digitales "regula el whistleblowing desde la perspectiva del derecho a la protección de datos personales" ${ }^{37}$ y contempla en su artículo 24 el derecho de los empleados y terceros a ser informados acerca de la existencia de dichos sistemas de información. Asimismo, la Guía de protección de datos en las relaciones laborales de la Agencia Española de Protección de Datos recuerda "que tanto los denunciantes como los potenciales denunciados deberán ser informados previamente a la existencia del sistema de denuncias internas, del tratamiento de los datos que conlleva la formulación de la denuncia y de las consecuencias que para el denunciado puede comportar el hecho de se denunciado". ${ }^{38}$

\subsection{Las novedades introducidas por la Directiva (UE) 2019/1937}

La gran novedad legislativa en esta materia llega de la mano de la Directiva (UE) 2019/1937 cuyo principal objetivo es, en la misma línea de los instrumentos anteriormente citados, garantizar la protección de los denunciantes frente a cualquier tipo de represalias derivadas del uso del canal de whistleblowing, en cualquiera de sus modalidades (denuncias internas, denuncias externas o revelación pública), ${ }^{39}$ puesto que

\footnotetext{
35 ESPÍN, Rosa, El Canal de Denuncias Internas en la Actividad Empresarial como Instrumento del Compliance (Tesis Doctoral). Universitat Autònoma de Barcelona, 2017. P. 149 y 154. Disponible en: (https://www.tdx.cat/bitstream/handle/10803/458436/roes1de1.pdf?sequence=1\&isAllowed=y)

${ }^{36}$ LOUSADA AROCHENA, José Fernando, op. cit., p. 3

${ }^{37}$ LOUSADA AROCHENA, José Fernando, op. cit., p. 4

${ }^{38}$ LOUSADA AROCHENA, José Fernando, op. cit., p. 8

${ }^{39}$ Considerando 45 de la Directiva (UE) 2019/1937
} 
un procedimiento inadecuado que no evitara represalias "sería totalmente ineficaz a los efectos de garantizar la actuación de la empresa dentro de la legalidad". ${ }^{40}$

Sin embargo, el contenido de la Directiva va más allá de la citada protección e incorpora distintas obligaciones para las empresas, especialmente en términos de investigación y vigilancia, que deberán encajarse con la doctrina actual en materia de protección del derecho a la intimidad, como se analizará a continuación.

Por un lado, la Directiva (UE) 2019/1937 impone la obligación de tener un canal de denuncias a las empresas que tengan 50 o más trabajadores (artículo 8.3) ${ }^{41}$. Por otro, se establece un plazo máximo de siete días para comunicar al denunciante la recepción de su denuncia (artículo 9.1.b.) y un plazo máximo de tres meses a partir del acuse de recibo (o seis meses en los casos debidamente justificados) para dar respuesta al denunciante (artículo 9.1.f.) comunicándole el resultado final de la investigación (artículo 11.1.d.).

Por lo tanto, a raíz de esta nueva regulación no solo se impone a las empresas la obligación de disponer de un canal de denuncia (para aquellas que superen el umbral fijado) sino la obligación de llevar a cabo una investigación interna en la empresa a partir de la recepción de una denuncia que permita, en un plazo temporal limitado, dar respuesta al denunciante sobre los resultados obtenidos.

Para desarrollar dicha investigación, se requerirá acceder a la información que permita obtener las respuestas necesarias y para ello, muy probablemente, resultará clave el acceso a datos que pudieran encontrarse en los medios tecnológicos de la empresa, tales como correo electrónico o cámaras de videovigilancia. Por lo tanto, las investigaciones internas de las empresas, en cumplimiento de las exigencias de la nueva Directiva (UE) 2019/1937 toparán con la obligación de respeto al derecho a la intimidad y vida privada de los trabajadores.

Esta situación conlleva dos consecuencias claras:

- La vigilancia y control que hasta ahora se definían como una facultad o potestad empresarial en virtud del artículo 20 ET, podrían convertirse en una obligación para el empresario.

- La existencia de una obligación de investigar junto con la obligación empresarial de respetar la intimidad y la vida privada de los trabajadores en el desarrollo de dichas

\footnotetext{
${ }^{40}$ LOUSADA AROCHENA, José Fernando, La protección laboral frente a represalias por la denuncia de irregularidades en la empresa (whistleblowing), Albacete, Editorial Bomarzo, 2020, p.30.

${ }^{41}$ Sin perjuicio de las exenciones contempladas en el artículo 8.9 de la Directiva (UE) 2019/1937
} 
funciones de vigilancia presentan un nuevo escenario de yuxtaposición de obligaciones. Ya no estamos ante la colisión de intereses empresariales con la obligación de respeto de derechos fundamentales, sino ante colisión de dos obligaciones: el deber de cumplir con la Directiva (UE) 2019/1937 y el deber de garantizar la intimidad y vida privada de los trabajadores.

Así las cosas, tal y como se analizará en el siguiente apartado, corresponderá determinar cuáles son los criterios que deben regir las investigaciones de las empresas iniciadas a raíz de la recepción de una denuncia interna y si, en su caso, el hecho de que exista una “obligación de investigar" permite relajar las exigencias jurisprudenciales en materia del derecho fundamental a la intimidad y a la vida privada de los trabajadores.

\subsection{La calificación de las denuncias internas}

En las distintas etapas o posiciones jurisprudenciales adoptadas por los tribunales en materia de protección del derecho fundamental a la intimidad y su ponderación respecto al poder de control empresarial, resulta común el papel o relevancia que tienen las sospechas del empresario ante la existencia de una posible conducta irregular en la empresa (tal y como se ha expuesto en el apartado 2).

Así se observa con las resoluciones del Tribunal Constitucional, como la STC 189/2000 (caso Ensidesa), que legitimó la injerencia empresarial a raíz de las irregularidades percibidas por el empresario, lo que a su vez justificó la diferencia en el fallo respecto de la SSTC 98/200 (caso La Toja). Del mismo modo ocurre con la STC 39/2016 (caso Bershka) y la doctrina derivada de la misma. Recientemente, además, así lo ha enfatizado el Tribunal Europeo de Derechos Humanos en la sentencia de la Gran Sala del caso López Ribalda II.

Aplicando dicha lógica al supuesto de una investigación iniciada a raíz de la recepción de una denuncia interna a través de un canal de whistleblowing, se suscita la siguiente cuestión: ¿La recepción de una denuncia interna, merece automáticamente la consideración de sospecha fundada? En función de la respuesta a esta pregunta, se plantean dos posibles escenarios.

En primer lugar, en caso de optar por una interpretación extensiva y entender que toda denuncia interna merece el tratamiento de sospecha fundada por parte del empresario, la imperativa investigación a la que obliga la normativa comunitaria podría llevarse a cabo aplicando la flexibilidad que la doctrina actual ofrece al empresario, principalmente según lo dispuesto en López Ribalda II. Por lo tanto, la investigación empresarial debería regirse por el principio de proporcionalidad, pero cabría emplear medidas que consistieran en 
una injerencia desinformada, en tanto que quedarían legitimadas por los motivos que rigen la actuación empresarial, esto es, la existencia de sospechas fundadas.

Los riesgos o amenazas asociados a esta interpretación derivarían de la posible existencia de denuncias falsas o infundadas debido al carácter abierto y potencialmente anónimo del canal $^{42}$ y la protección que la Directiva (UE) 2019/1937 otorga al trabajador frente a posibles represalias. Asimismo, una posición tan expansiva podría ofrecer al empresario una vía de legitimación genérica (ante cualquier queja o denuncia, sin valorar su veracidad) para justificar una mayor injerencia en las investigaciones realizadas.

En segundo lugar, en el supuesto de entender que la denuncia interna recibida por la empresa no merece la automática elevación a la categoría de sospecha fundada, la investigación empresarial podría dividirse en dos fases:

- De entrada, una primera etapa en la investigación debería consistir en analizar los detalles de la denuncia. En esta primera fase, centrada en determinar la veracidad de la denuncia, debería aplicarse la doctrina propia de los supuestos en los que no existen sospechas empresariales (doctrina Barbulescu II). Es decir, el requisito de la información previa adoptaría una mayor rigidez y la opacidad empresarial en sus actuaciones de investigación estaría sometida a mayores exigencias para superar el juicio de proporcionalidad. En este punto, se plantearía una nueva cuestión, relativa a cuál debería ser la información proporcionada a los trabajadores en este punto de la investigación o si, por ejemplo, bastaría con informar a los representantes de los trabajadores (garantizando la transparencia, así como la confidencialidad de la investigación por si se concluye que las sospechas o irregularidades que se plantean en la denuncia son fundadas).

- Una vez alcanzada una respuesta a la pregunta anterior, se iniciaría una segunda etapa en la investigación de la empresa, en la que posiblemente podrían encontrarse tres escenarios:

a. A raíz de las comprobaciones realizadas, se concluye que la denuncia es falsa e infundada, de modo que se da la investigación por terminada y se llevan a cabo las oportunas actuaciones respecto al falso denunciante.

b. No puede concluirse que existan sospechas fundadas sobre la existencia de una irregularidad en la empresa, pero, aun así, se decide continuar investigando. En este caso, la investigación se debería regir por los mismos estándares que los aplicables para la primera etapa.

\footnotetext{
${ }^{42}$ La Directiva (UE) 2019/1937 deja al arbitrio de los Estados Miembros en el momento de transposición de su contenido la decisión sobre si las denuncias a través de canales internos pueden ser o no anónimas.
} 
c. Tras la primera fase de investigación, se considera que la denuncia recibida tiene consideración de sospecha fundada y, en consecuencia, se continua la investigación pudiendo aplicar unos estándares y criterios más flexibles, de acuerdo con lo dispuesto por el Tribunal Europeo de Derechos Humanos en López Ribalda II.

Dichos escenarios, si bien hipotéticos por el momento, probablemente se planteen con mayor fuerza en el momento en que la Directiva (UE) 2019/1937 sea transpuesta en la normativa española. Por ello, sería interesante que, en el ejercicio de dicha transposición, el legislador español contemplara las diferentes situaciones en las que podría encontrarse el empresario a raíz de esta nueva normativa. De lo contrario, pueden derivarse muchas dudas y una gran inseguridad jurídica respecto a la forma de conciliar la yuxtaposición de obligaciones existentes en esta materia: i) la obligación de llevar a cabo una investigación interna y dar respuesta en el tiempo requerido a la denuncia recibida junto con ii) la obligación de respetar el derecho fundamental a la intimidad y vida privada de los trabajadores investigados.

\subsection{La responsabilidad penal de la persona jurídica ante la omisión de vigilancia y control}

Por último, conviene resaltar una tercera obligación o riesgo empresarial que podría entrar en juego en esta materia: la posibilidad de la empresa de incurrir responsabilidad penal. A raíz de la modificación del artículo 31 bis del Código Penal en el año $2010^{43}$ se introdujo en el ordenamiento nacional la posibilidad de que las personas jurídicas sean susceptibles de cometer delitos y, por lo tanto, de incurrir en responsabilidad penal. Dicho artículo, en su apartado segundo, fue modificado en $2015,{ }^{44}$ estableciendo que las personas jurídicas quedarán exentas de responsabilidad penal cuando, con carácter preventivo, hubieren implementado "las medidas de vigilancia y control idóneas para prevenir delitos de la misma naturaleza o para reducir de forma significativa el riesgo de su comisión". 45

Es precisamente como consecuencia de este cambio legislativo que, en los últimos años, las empresas han dedicado importantes esfuerzos a desarrollar programas de compliance

\footnotetext{
${ }^{43}$ Ley Orgánica 5/2010, de 22 de junio, por la que se modifica la Ley Orgánica 10/1995, de 23 de noviembre, del Código Penal (“LO 5/2010”).

44 Ley Orgánica 1/2015, de 30 de marzo, por la que se modifica la Ley Orgánica 10/1995, de 23 de noviembre, del Código Penal (“LO 1/2015”).

${ }^{45}$ Artículo 31 bis, apartado segundo LO 1/2015.
} 
o cumplimiento normativo, entre los cuales se encuentran, como se ha expuesto, los canales de denuncia.

Por lo tanto, a la doble obligación impuesta al empresario en el ámbito laboral, se une el riesgo de poder incurrir en la comisión de un delito en calidad de persona jurídica en caso de que se produzca en el seno de la organización un delito, y que la empresa no pueda demostrar haber implementado todas las medidas de investigación y control pertinentes para considerar su actuación como diligente.

Al unir los tres vértices de estas obligaciones, se plantearía una nueva cuestión: ¿qué obligación debe imperar en aquellos supuestos en los que la denuncia interna alerte de la comisión en la empresa de un delito castigado por la normativa penal, cuyas consecuencias podrían conllevar la responsabilidad penal de la persona jurídica? Es decir, volviendo a la casilla de salida del escenario que planteábamos en el punto anterior, cabe preguntarse si el hecho de que la denuncia interna alerte sobre la posible comisión de un delito permitiría calificar a la denuncia interna automáticamente como sospecha fundada, con las consiguientes prerrogativas que dicha calificación otorgaría al empresario en el desarrollo de la investigación interna. Investigación que, en este segundo supuesto, no sería únicamente imperativa con base en la Directiva (UE) 2019/1937, sino también según lo establecido en el artículo 31 bis Código Penal.

Por todo lo expuesto, la posible concurrencia de hasta tres obligaciones empresariales existentes en esta materia requiere una respuesta integrada que permita homogeneizar la actuación en cada uno de estos campos e impedir que, en cumplimiento de una de las obligaciones, se esté incurriendo en una vulneración de otra. De lo contrario, cabría esperar una actuación empresarial guiada por un criterio económico o reputacional, es decir, priorizando el cumplimiento de aquellas obligaciones cuya infracción pudiera resultar más costosa para la organización.

\section{Conclusiones}

El respeto a la intimidad y a la vida privada de los trabajadores, como derecho fundamental reconocido en el artículo $8 \mathrm{CEDH}$ y $18 \mathrm{CE}$, ha sido objeto de una gran evolución y desarrollo normativo en las últimas décadas. Principalmente, a partir de la incorporación masiva de medios tecnológicos y electrónicos en el lugar de trabajo, que ha traído consigo grandes cambios en la forma de ejercer la facultad de control empresarial.

Debido a la inactividad legislativa en la materia, los tribunales han venido dictando los estándares y criterios que deben regir la actuación empresarial, en ejercicio de una suerte 
de stare decisis donde los precedentes han sido la única guía tanto para tribunales como sujetos privados.

En la actualidad, la doctrina aplicable en materia de protección de la intimidad y privacidad de los trabajadores viene principalmente determinada por las resoluciones del Tribunal Europeo de Derechos Humanos Barbulescu II y López Ribalda II, que sientan los criterios (seis) que deben seguirse para valorar la legalidad de la injerencia empresarial realizada, basados en la aplicación de los principios de proporcionalidad de la medida e información previa a los trabajadores. Además, conforme al posicionamiento más reciente de la Gran Sala (López Ribalda II), podrán admitirse medidas empresariales desinformadas en casos donde la viabilidad de la medida, por la existencia de sospechas fundadas y la inexistencia de medidas alternativas para obtener el resultado, las hicieran necesarias.

Dichos precedentes, han sido tradicionalmente analizados desde la perspectiva y en contraposición con el artículo 20.3 ET, que reconoce la facultad o poder de control empresarial. Sin embargo, la introducción de la Directiva (UE) 2019/1937 y el artículo 31 bis del Código Penal traen consigo la incorporación de la obligación empresarial de investigar en el seno de la organización, modificando el prisma desde el cual deberán ponderarse las injerencias realizadas a la vida privada de los trabajadores.

Así, ante el recibimiento de una denuncia interna a través de un canal de whistleblowing, el deber empresarial de investigar que impone la Directiva (UE) 2019/1937 puede conllevar i) la conversión de la facultad de control prevista en el artículo 20.3 ET en una obligación y ii) la existencia de una yuxtaposición de obligaciones, junto con el deber de protección del derecho fundamental de los trabajadores a la intimidad. Del mismo modo, el artículo 31 bis del Código Penal prevé iii) la posibilidad por parte de la persona jurídica de incurrir en responsabilidad penal ante la falta de control o vigilancia suficiente para prevenir la comisión de delitos en la empresa.

Las novedades que necesariamente se derivan de la introducción de dichos instrumentos normativos requiere dar respuesta a múltiples cuestiones sobre cuál debería ser la adecuada conjugación de los criterios existentes en materia de protección del derecho a la intimidad y a la vida privada de los trabajadores, y si éstos podrían flexibilizarse ante el recibimiento de una denuncia interna en la empresa -si merecen o no la calificación como sospechas fundadas a efectos de la investigación-. Más todavía, cuando las investigaciones internas iniciadas a raíz del recibimiento de una denuncia a través de un canal de whistleblowing, pueden plantear hasta una triple colisión de obligaciones para el empresario, previstas en fuentes normativas distintas. 
La ausencia de una respuesta integrada ante dicho escenario plantea un contexto de inseguridad jurídica para los distintos actores intervinientes. Por ello, es necesaria la concreción de criterios que arrojen luz sobre la forma de proceder y de realizar las investigaciones internas en las empresas, en las que la confluencia de obligaciones plantea dudas $\mathrm{y}$, sobre todo, con el fin de dar una respuesta adecuada a las necesidades de i) investigación, ii) prevención de delitos y iii) protección de derechos fundamentales de los trabajadores.

\section{Bibliografía}

BLASCO JOVER, Carolina, "Trabajadores "transparentes": la facultad fiscalizadora del empresario vs derechos fundamentales de los empleados (I)", Revista Internacional y Comparada de Relaciones Laborales y Derecho del Empleo, Adapt University Press, 2018 pp. 29-56. Disponible en: http://ejcls.adapt.it/index.php/rlde_adapt/article/view/600.

ESPÍN, Rosa, "El Canal de Denuncias Internas en la Actividad Empresarial como Instrumento del Compliance" (Tesis Doctoral). Universitat Autònoma de Barcelona, 2017.

en: https://www.tdx.cat/bitstream/handle/10803/458436/roes1de1.pdf?sequence=1\&isAllo wed $=\mathrm{y}$.

FLANAGAN, Julie A., "Restricting electronic monitoring in the private workplace" Duke Law Journal, Vol. 93, 1994, pp. 1256-1281. Disponible en: https://scholarship.law.duke.edu/dlj/vol43/iss6/6/.

GARCÍA RUBIO, Ma Amparo, "Nueva doctrina constitucional sobre videovigilancia y protección de datos personales", ELDERECHO.COM, 2016. Disponible en: https://elderecho.com/nueva-doctrina-constitucional-sobre-videovigilancia-laboral-yproteccion-de-datos-personales [Última consulta 4 de julio de 2020].

HENRÍQUEZ TRILLERÍA, Sebastián, "Protección de datos, videovigilancia laboral y doctrina de la Sentencia López Ribalda II: un peligroso camino hacia la degradación de la obligación de información" IUSLabor, núm. 3, 2019, pp. 59-62. Disponible en: https://www.upf.edu/web/iuslabor/3/2019.

LOUSADA AROCHENA, José Fernando, La protección laboral frente a represalias por la denuncia de irregularidades en la empresa (whistleblowing), Albacete, Editorial Bomarzo, 2020. 
LOUSADA AROCHENA, José Fernando, "Sistemas de denuncias internas (Whistleblowing) y derechos fundamentales en el trabajo", Trabajo y Derecho, núm. 52/2019, pp. 24 a 36.

MARTÍN POLVORINOS, César, "Guía de implementación de compliance para Pymes". World Compliance Association, 2019, pp. 109 a 112. Disponible en: http://www.worldcomplianceassociation.com/documentacion/Guia_Compliance_web_v .02.pdf.

MARTINEZ FONS, Daniel, "El poder de control del empresario en la relación laboral". Madrid, Consejo Económico Social, 2002.

MERCADER UGUINA, Jesús, "El impacto del uso de dispositivos tecnológicos y Derechos Fundamentales: nuevas respuestas para un tiempo nuevo", IUSLabor, núm. 2, 2018. Disponible en: https://www.upf.edu/web/iuslabor/2/2018.

PÉREZ TREVIÑO, José Luís, "Whistleblowing”, Eunomía. Revista en cultura de la legalidad, núm. 14, 2018, pp. 285-298.

RODRÍGUEZ ESCANCIANO, Susana, "Poder de control empresarial, sistemas tecnológicos y derechos fundamentales de los trabajadores". Tirant lo Blanch, Valencia, 2015.

SALA FRANCO, Tomás, "El derecho a la intimidad y a la propia imagen y las nuevas tecnologías de control laboral" en BORRAJO DACRUZ, EFRÉN (coord.) Trabajo y libertades públicas, Madrid, Wolkers Kluwer (La Ley), 1999.

SEMPERE NAVARRO, Antonio Vicente, "Un apunte sobre la grabación mediante cámaras (Al hilo de la STS-CIV 600/2019 de 7 de noviembre)", Revista Aranzadi Doctrinal, núm. 2, 2020. 